\title{
Feeding a Black Hole Jet
}

\author{
Simulations of the environment around a spinning black hole give new insight into the \\ formation of luminous jets seen from Earth.
}

\section{by Robert F. Penna*}

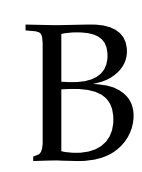
lack holes are creatures of darkness. They creep through the Universe emitting no light of their own, outside of a faint halo of Hawking radiation that is all but invisible for known black holes. So it is one of the great ironies of astronomy that, time and again, black holes have been found lurking in the brightest places in the cosmos. This association between black holes and light arises because the incredible gravitational forces exerted by a hole can impart energy to nearby material, causing it to radiate. An example of this process occurs in black hole jets, where black hole rotation and magnetic fields combine to create a stream of plasma particles that emit light over a wide range of wavelengths. A new set of simulations-performed by Kyle Parfrey from Lawrence Berkeley National Laboratory, California, and colleagues-offers an in-depth look at black hole jets [1], tracking, for the first time, the motion of

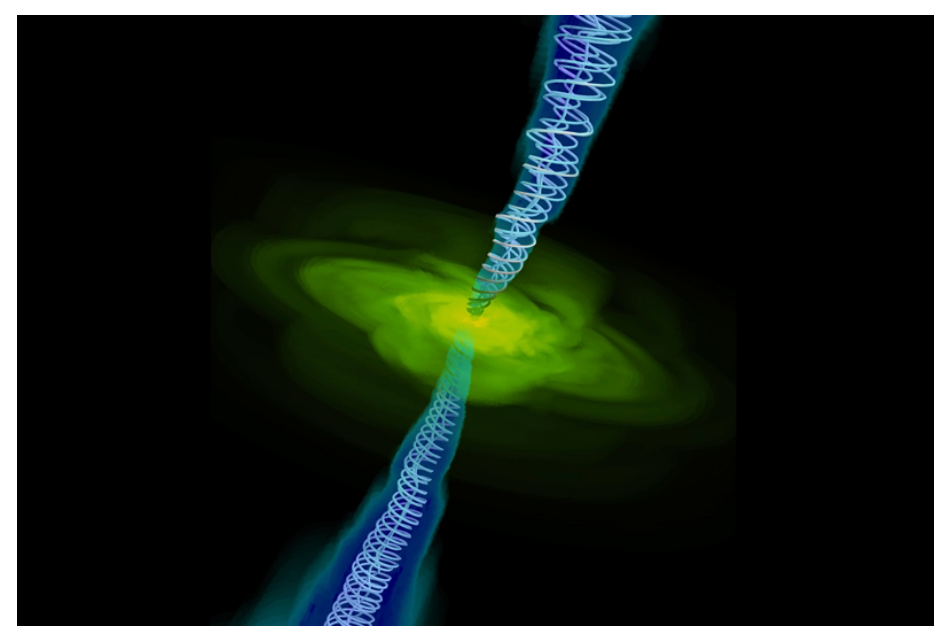

Figure 1: An artist's depiction of a black hole with two opposing jets. The black hole is surrounded by a cloud of infalling gas (green), while the jets are defined by spiraling magnetic field lines (blue). (Alexander Tchekhovskoy/Lawrence Berkeley National Laboratory)

*Center for Theoretical Physics, Columbia University, New York, NY, USA the plasma particles that are produced through pair creation in the vicinity of the black hole. The results show that large numbers of these particles rob energy from the black hole's rotation. These kinds of simulations may play a key role in decoding the signals from black holes in the distant Universe and in our own Milky Way.

To build a black hole jet, nature has a tried-and-true formula. Start with a black hole that is spinning around so fast that its event horizon-the surface separating the inside of the black hole from the outside-is racing around at an appreciable fraction of the speed of light. Now thread the spinning black hole with a magnetic field. Calculations show that the black hole's rotation, along with the effect of infalling gas, will cause the magnetic field lines to wind around into giant helixes that spiral out along the black hole's rotational axis (Fig. 1). In this process, the black hole's rotational energy is slowly transferred to the magnetic field. The energy in the magnetic field is eventually dissipated and converted to ordinary radiation through a cascade process involving pair creation and synchrotron effects. Black hole jets such as this are believed to power some of the brightest sources of $x$-ray and radio emission in the sky [2].

Over the past decade, there have been many efforts to understand black hole jets using numerical simulations. Current state-of-the-art simulations use the theory of general relativistic magnetohydrodynamics (GRMHD), which describes the motion of a magnetized fluid in curved spacetime [3]. These simulations are very good at describing the transfer of energy from the spinning black hole to the magnetic field. However, they treat the plasma as a continuous fluid, and they set the density "by hand" with a somewhat ad hoc value. In real astrophysical jets, the plasma is so rarefied that the fluid approximation is not really valid, and its density is constantly adjusting to local interactions between plasma particles and the magnetic field. Because of their limitations, GRMHD simulations are unable to model the conversion of magnetic-field energy into ordinary radiation, and they have yet to resolve lingering questions about the overall strength of the jets.

To tackle these problems, Parfrey et al. have introduced a new kind of black hole jet simulation. In their work, the plasma is described correctly as a collection of individual particles, rather than as a smooth, continuous fluid. The density of the plasma is still not based on a first-principles model of the interactions between the particles and the 
magnetic field, but Parfrey et al. use a physically motivated prescription for setting the plasma density, based on electron-positron pair creation in the electric field that is induced by magnetic-field dynamics around the black hole.

Like GRMHD simulations, the new simulations require a lot of computing power to observe small-scale spatial variations in the jet. As such, the Parfrey et al. paper presents only two high-resolution runs, both of which describe black holes rotating at about $96 \%$ of the speed of light. In one run, the threshold for pair creation is set low, leading to a highdensity plasma. In the other run, the threshold is higher, and the resulting plasma is less dense. The final results in both cases are short movies, each lasting just long enough for the black hole to spin around about a dozen times. Despite the brevity of the simulations, the jets appear to relax to a quasisteady state about midway through the runs.

The results of the new simulations are not radically different from the those of the old GRMHD simulations, which is, in some sense, reassuring. However, Parfrey et al. uncover some interesting and novel behavior. For example, they find a large population of particles whose relativistic energies are negative, as measured by an observer far from the black hole. When these particles fall into the black hole, the black hole's total energy decreases (see 26 June 2015 Focus Story). The possibility of creating negative-energy particles near a rotating black hole was predicted long ago by Roger Penrose [4]. What is surprising is that the new simulations show a substantial flow of these particles into the black hole, so much so that the energy they extract by falling into the hole is comparable to the energy extracted by the winding of the magnetic field. Follow-up work is needed to confirm this prediction, but if the effect of negative-energy particles is as strong as claimed, it could alter expectations for the radiation spectra from black hole jets.

The years ahead are full of promise. Gravitational-wave detectors have begun to pick up the ripples in spacetime left behind by the mergers of distant black holes [5]. Although not yet seen, black holes should also emit gravitational waves when they swallow a star. These gravitational waves should arrive accompanied by ordinary light. Jet models like the ones developed by Parfrey et al. will play a key role in interpreting these observations. Closer to home, the Event Horizon Telescope (EHT) is poised to provide the highestresolution look yet at the black hole lurking at the center of our Galaxy [6]. This black hole has a modest jet, so EHT data will need to be processed with help from realistic jet models. Like black hole environments, the future is bright for black hole research.

This research is published in Physical Review Letters.

\section{REFERENCES}

[1] K. Parfrey, A. Philippov, and B. Cerutti, "First-principles plasma simulations of black-hole jet launching," Phys. Rev. Lett. 122, 035101 (2019).

[2] R. D. Blandford and R. L. Znajek, "Electromagnetic extraction of energy from Kerr black holes," Mon. Not. R. Astron. Soc. 179, 433 (1977).

[3] C. F. Gammie, J. C. McKinney, and G. Tóth, "HARM: A numerical scheme for general relativistic magnetohydrodynamics," Astrophys. J. 589, 444 (2003).

[4] R. Penrose and R. M. Floyd, "Extraction of rotational energy from a black hole," Nature Phys. Sci. 229, 177 (1971).

[5] B. P. Abbott et al., "Observation of gravitational waves from a binary black hole merger," Phys. Rev. Lett. 116, 061102 (2016).

[6] H. Falcke, F. Melia, and E. Agol, "Viewing the shadow of the black hole at the galactic center," Astrophys. J. Lett. 528, L13 (1999).

10.1103/Physics. 12.5 